\title{
Analysis of subspace-based direction of arrival estimation methods
}

\author{
BHASKAR D RAO and K V S HARI \\ AMES Department - System Science, University of California, San Diego, \\ La Jolla, California 92093-0411, USA
}

\begin{abstract}
In this paper, a general framework for the analysis of eigenbased subspace methods is developed. It is shown that a two-step procedure can be effectively used to analyse subspace methods under fairly general conditions. The first step relates the errors in the covariance matrix to errors in the subspaces, and the second step relates error in the subspaces to the errors in the direction of arrival (DOA) estimates. Combining these two steps along with the statistics of the data, expressions for the mean squared error in the DOA estimate are derived. The potential of the approach is demonstrated by analysing two subspace methods, MUSIC and the minimum-norm method.
\end{abstract}

Keywords. Array processing; direction of arrival estimation; subspace methods; statistical analysis.

\section{Introduction}

Eigen-decomposition based methods have recently received a great deal of attention (Bienvenu \& Kopp 1979; Schmidt 1979; Kumaresan \& Tufts 1983; Cadzow 1988; Rao 1988; Ottersten 1989; Roy \& Kailath 1989; Viberg 1989). These methods, referred to as subspace methods, decompose the space spanned by the eigenvectors of the observed covariance matrix into two orthogonal subspaces referred to as the signal and noise subspace. In the recent past, an extensive analysis of these methods has been conducted by a number of researchers providing valuable insight. The first work in this context has been presented in Jeffries \& Farrier (1985) and Kaveh \& Barabell (1986), where the resolution capability of MUSIC and the Minimum-Norm method were studied by examining the null spectrum. Recently, expressions for the asymptotic mean squared error in the direction of arrival (DOA) estimate have been developed (Porat \& Friedlander 1988; Rao \& Hari 1989a, b; Stoica \& Nehorai 1989, 1991). The results have also been extended to deal with the effect of spatial smoothing (Clergeot et al 1989; Pillai \& Kwon 1989; Rao \& Hari 1990) and sensor errors (Friedlander 1989; Swindlehurst et al 1989). Using the insight gained from these results, a general framework for analysing subspace methods is developed. The overall 
approach consists of two main steps. The first step establishes a relationship between perturbation in the covariance estimate and the estimated subspaces. The second step consists of relating the errors in the subspaces to the error in the DOA. Combining the two steps along with the statistics of the data, the methods can be statistically characterized. In this paper, it is shown how this framework can be used beneficially by analysing subspace methods like MUSIC and the minimum-norm methods under fairly general conditions.

\section{Problem formulation}

\subsection{Data model}

The problem of estimating the DOA of $M$, possibly coherent signals impinging on a uniform linear array (ULA) of $L_{1}$ sensors is considered. For the $n$th observation period (snapshot), the spatial samples of the signal plus noise are given by the output vector

$$
\begin{aligned}
& Y(n)=\left[y_{1}(n), y_{2}(n), \ldots, y_{L_{1}}(n)\right]^{T} \\
& =\left[\sum_{i=1}^{M} p_{i}(n), \sum_{i=1}^{M} p_{i}(n) e^{j \omega_{1}}, \ldots, \sum_{i=1}^{M} p_{i}(n) e^{j\left(L_{1}-1\right) \omega_{i}}\right]^{T}+N(n) \\
& =\left[\begin{array}{cccc}
1 & 1 & & 1 \\
e^{j \omega_{1}} & e^{j \omega_{2}} & & e^{j \omega_{M}} \\
\vdots & \vdots & \cdots & \vdots \\
e^{j\left(L_{1}-1\right) \omega_{1}} & e^{j\left(L_{1}-1\right) \omega_{2}} & & e^{j\left(L_{1}-1\right) \omega_{M}}
\end{array}\right]\left[\begin{array}{c}
p_{1}(n) \\
p_{2}(n) \\
\vdots \\
p_{M}(n)
\end{array}\right]+N(n) \\
& =V_{s} p(n)+N(n)
\end{aligned}
$$

where $\omega_{i}=\left(2 \pi d / \lambda_{0}\right) \sin \theta_{i}, d$ being the separation between sensors, $\lambda_{0}$ the wavelength of the incident signal, and $\theta_{i}$ (or $\omega_{i}$ ) the DOA. $V_{s}$ is the array steering matrix for the ULA and each column of $V_{s}$ is known as the array steering vector. The following assumptions are made regarding the data ${ }^{1}$. The covariance matrix of the source amplitudes is $P$ where $P=\overline{p(n) p^{H}(n)}$. Also the noise vector $N(n)$ is assumed to be zero mean, white and independent of the source amplitudes $p(n)$. Hence,

$$
R=\overline{Y(n) Y^{\bar{H}}(n)}=V_{s} P V_{s}^{H}+\sigma_{n}^{2} I=R_{s}+\sigma_{n}^{2} I .
$$

The basic idea behind the subspace methods is to recover the subspace spanned by the columns of $V_{s}$, referred to as the signal subspace, and use it to estimate the DOA $\omega_{i}$.

\subsection{Subspace decomposition}

One approach to recovering the signal subspace is through the eigendecomposition of the data covariance matrix. Given the exact covariance matrix, its eigen-

\footnotetext{
"In this paper, the overbar "—" will be used to denote the expectation operator. The superscript $T$ is used to denote transpose, ${ }^{*}$ to denote complex conjugate, $H$ to denote complex conjugate transpose, and ${ }^{+}$to denote the pseudoinverse.
} 
decomposition is given by ${ }^{2}$

$$
R=\sum_{l=1}^{l} \lambda_{l} S_{l} S_{l}^{H}=E \Lambda E^{H}=E_{s} \Lambda_{s} E_{s}^{H}+\sigma_{n}^{2} I=R_{s}+\sigma_{n}^{2} I,
$$

where

Also

$$
\begin{aligned}
& E=\left[S_{1}, S_{2}, \ldots, S_{L}\right], \quad E_{s}=\left[S_{1}, S_{2}, \ldots, S_{M}\right], \quad E_{n}=\left[S_{M+1}, S_{M+2}, \ldots, S_{L}\right] \\
& \Lambda=\operatorname{diag}\left(\lambda_{1}, \lambda_{2}, \ldots, \lambda_{L}\right), \quad \text { and } \Lambda_{s}=\operatorname{diag}\left(\lambda_{1}^{s}, \lambda_{2}^{s}, \ldots, \lambda_{M}^{s}\right)
\end{aligned}
$$

$$
\begin{aligned}
\lambda_{1}=\lambda_{1}^{s}+\sigma_{n}^{2} \geqslant \lambda_{2} & =\lambda_{2}^{s}+\sigma_{n}^{2} \geqslant \ldots \\
& \geqslant \lambda_{M}=\lambda_{M}^{s}+\sigma_{n}^{2}>\lambda_{M+1}=\cdots=\lambda_{L}=\sigma_{n}^{2} .
\end{aligned}
$$

$\lambda_{i}$ are the eigenvalues of $R$, and $S_{i}$ the corresponding orthonormal eigenvectors. Since the first $M$ eigenvectors $\left(E_{s}\right)$ of $R$ are the same as those of $R_{s}$, the noise free covariance matrix, the range space of $E_{s}$ is the same as that of $V_{s}$. The subspace spanned by the columns of $E_{n}$ is orthogonal to the signal subspace, and is often referred to as the noise subspace.

It is useful to note that the important feature of $R$ that is exploited by the subspace methods is the structure in its signal subspace. Other covariance matrices can be constructed from the data $Y(n)$ with properties similar to $R$ in (1), i.e. their signal subspace is structured and has dimension $M$. Popular alternate covariance matrices are the forward only, backward only, forward smoothing and forward backward smoothing covariance matrices (Shan et al 1985; Williams et al 1988). Though these covariance matrices gives rise to a different $R_{s}$, they share an important property that the range space of $R_{s}$ is equal to the range space of $V_{s}$.

\section{Performance analysis}

\subsection{Framework for analysis}

In practice, the exact covariance matrix is not available and so an estimate is often used. It is easy to observe that if the covariance matrix is perturbed, the subspaces are perturbed which results in errors in parameter estimates as illustrated below ${ }^{3}$.

$$
Y(n) \stackrel{h}{\rightarrow}(R+\Delta R) \stackrel{g}{\rightarrow}(E+\Delta E) \stackrel{f}{\rightarrow}\left(\omega_{i}+\Delta \omega_{i}\right)
$$

$f, g, h$ are some deterministic mapping functions. $h$ denotes the mapping between the data and the covariance estimate, $g$, the mapping between $R$ and the eigenvectors, and $f$ depends upon the subspace method used to estimate the DOA $\omega_{i}$. The procedure for analysis can be divided into the following steps.

1. The first step is to determine the relationship $(g)$ between the perturbed covariance matrix and the perturbed subspaces.

2. The second step is to determine a relation between the perturbation in the subspaces and the error in the DOA estimates. This depends on the subspace method being analysed.

\footnotetext{
${ }^{2}$ Subscripts $s$ and $n$ are used to denote parameters associated with the signal and noise respectively.

${ }^{3}$ Prefix $\Delta$ represents the error in the quantity.
} 
3. Combining the two steps along with the statistics of the covariance matrix, expressions for the mean squared error in the DOA estimates can be derived.

\subsection{Perturbation in the subspaces}

Given an estimate ${ }^{4}$ of the covariance matrix $\hat{R}$, its eigenvectors and hence the subspaces are in error. Starting from the statistics of the eigenvectors, the errors in the DOA estimates can be determined as was done for the forward only case (Kaveh \& Barabell 1986; Jeffries \& Farrier 1988; Porat \& Friedlander 1988; Stoica \& Nehorai 1989; Rao \& Hari 1989b). However, the approach based on characterizing the error (first order) in the subspaces due to $\Delta R$ directly, is more effective. For this purpose it is useful to note that given an estimate of the covariance matrix, the errors in the estimated eigenvectors contributing to the error in the DOA estimates, are orthogonal to the subspace under consideration. For example, in the case of noise subspace methods, if $\Delta E_{n}$ represents the error in the noise eigenvectors, only $P_{s} \Delta E_{n}$, the signal subspace component of the estimated noise eigenvectors, is responsible for errors in the DOA estimates (Clergeot et al 1989; Hari 1990; Rao \& Hari 1990b). This observation greatly enhances the tractability of the analysis as will be seen from the results presented. An expression for these errors are summarized in the following theorem.

Theorem 1. (Clergeot et al 1989; Hari 1990; Rao \& Hari 1990) Assuming that the signal eigenvalues of $R$ are distinct, the first order perturbation of the error in the estimated signal eigenvectors projected onto the noise subspace and the error in the noise eigenvectors projected onto the signal subspace are given by

$$
\begin{aligned}
& P_{n} \Delta E_{s}=P_{n} \Delta R R_{s}^{+} E_{s}=P_{n} \hat{R} R_{s}^{+} E_{s} \\
& P_{s} \Delta E_{n}=-R_{s}^{+} P_{s} \Delta R E_{n}=-R_{s}^{+} P_{s} \hat{R} E_{n}
\end{aligned}
$$

where $P_{s}=\left(I-P_{n}\right)=E_{s} E_{s}^{H}, \hat{R}=R+\Delta R$ with $\widehat{R}=R, \hat{E}_{s}=E_{s}+\Delta E_{s}$, and $\hat{E}_{n}=E_{n}+\Delta E_{n}$.

Note that (2) and (3) require no statistical assumptions about $\hat{R}$. Hence, if properly applied, they can lead to fairly general results as indicated below.

The usefulness of the theorem lies in the fact that no assumption is made about the source of error in the covariance matrix. It could be due to noise in the data, sensor location errors, array calibration errors etc. Therefore theorem 1 provides a convenient tool to analyse the subspace methods with respect to various kinds of errors in the covariance matrix.

- The resulting first order perturbation equations for the DOA estimates are valid for any $N$. In the case of deterministic source amplitudes, i.e. $p(n)$ being deterministic, they can be used for all $N$, and for small values of $N$ are useful in predicting the performance of subspace methods under high SNR conditions. $N=1$ corresponds to the time series frequency estimation problem thereby providing a unified framework for the analysis of subspace based DOA estimation and time series frequency estimation methods (Rao \& Hari 1990a). Also, the resulting expressions for the frequency estimation problem are more accurate than the existing results which are derived using a first-order perturbation of the data matrix (Hua \& Sarkar 1988).

\footnotetext{
$4^{-}$is used to denote estimates.
} 
Theorem 1 can also be used for conducting an asymptotic (large $N$ ) analysis, and the resulting mean squared expressions are accurate to $o\left(N^{-1}\right)$. These asymptotic results depend on the asymptotic normality of $\sqrt{N}(\hat{R}-R)$ which can usually be proven using the multivariate central limit theorem (MCLT) under fairly weak conditions (Brillinger 1980). The asymptotic normality of the DOA estimates then follows from the asymptotic normality of $\hat{R}$ and by the use of theorem P5.2 in Brillinger (1980). Since the DOA estimates $\hat{\omega}_{i}$ are bounded between 0 and $2 \pi$, the asymptotic moments are also equal to the moments of the limiting distribution (Chung 1974; Hari 1990). Also, it turns out that except for the second-order properties of the source amplitudes, their exact distribution is not important for the asymptotic analysis of subspace based methods (Ottersten et al 1990; Rao \& Hari 1990a).

\subsection{Statistics}

To determine the mean squared error in the DOA estimates, some statistical assumptions have to be made regarding $\hat{R}$. However, to carry out the analysis these assumptions need not be made at this stage. It suffices to just define the following quantity $\Gamma_{\alpha \beta \gamma \delta}$ as ${ }^{5}$

$$
\Gamma_{\alpha \beta \gamma \delta}=\operatorname{Cov}\left[\left(\alpha^{H} \hat{R} \delta\right),\left(\beta^{H} \hat{R} \gamma\right)\right],
$$

with $\alpha, \beta, \gamma, \delta$ being vectors of appropriate dimensions. The mean squared error expressions are derived in terms of $\Gamma$. Clearly, in addition to the vectors $\alpha, \beta, \gamma, \delta$, $\Gamma_{\alpha \beta \gamma \delta}$ also depends on $\hat{R}$. Consequently, to study the effect of various errors in $R$, it is necessary to determine a more explicit expression for $\Gamma_{\alpha \beta \gamma \delta}$ under those conditions, and substitute for it in the mean squared error expressions. As indicated before, the perturbation in $R$ could be due to various sources of errors like array calibration errors, sensor location errors, finite number of snapshots and sensor noise. In this paper, first the general expressions for the mean squared error in the DOA estimate using MUSIC and the minimum-norm method are derived. Then the results are specialized to obtain asymptotic results for the forward only covariance estimator in the presence of additive white noise in the data.

\section{Analysis of MUSIC}

In spectral MUSIC, the DOA are found by locating the peaks of the spatial spectrum $S(\omega)=1 / D\left(e^{j \omega}\right)$, where $D_{m u}\left(e^{j \omega}\right)$, the null spectrum, is defined as ${ }^{6}$

$$
D\left(e^{j \omega)}\right)=V^{H}(\omega) P_{n} V(\omega)
$$

and $V(\omega)=\left(1 / \sqrt{L_{1}}\right)\left[1, e^{j \omega}, \cdots, e^{j\left(L_{1}-1\right) \omega}\right]^{T}$. Also $V_{1}(\omega)=\partial V(\omega) / \partial \omega$. The DOA can also be obtained using Root-Music for a uniform linear array (Rao \& Hari 1989b). The performance of the method is evaluated by examining the mean squared error $\overline{\left.\Delta z_{i}\right|^{2}}$ in the estimates of the signal zeros, and the mean squared error $\overline{\left(\Delta \omega_{i}\right)^{2}}$ in the DOA

\footnotetext{
${ }^{5} \operatorname{Cov}(X, Y)=\overline{(X-\bar{X})(Y-\bar{Y})^{H}}$.

${ }^{6}$ Subscript $m u$ denotes quantity associated with MUSIC.
} 
estimates. The motivation for examining $\overline{\left|\Delta z_{i}\right|^{2}}$ is that it is directly related to the bias in the null spectrum and provides insight into spectral forms. A quantitative way of comparing the root and spectral forms is through the spectral efficiency factor $\gamma$, where $\gamma=\overline{(\Delta \omega)^{2}} / \overline{\left.\Delta z\right|^{2}}$. If $\Delta \omega$ is measured in radians, then $0 \leqslant \gamma \leqslant 1$. A value of $\gamma$ close to zero implies that the spectral forms are less effective and a value close to one implies that both forms are equally effective $\left(z_{i}=e^{j \omega_{i}}\right)$.

\subsection{Mean squared error in the signal zeros}

From the analysis of MUSIC in Rao \& Hari (1989b), the mean squared error (MSE) in the signal zeros is given

$$
\overline{\left|\Delta z_{i}\right|_{m u}^{2}}=\bar{D}\left(e^{j \omega_{i}}\right) /\left[V_{1}^{H}\left(\omega_{i}\right) P_{n} V_{1}\left(\omega_{i}\right)\right]
$$

For notational convenience, we shall use $V$ and $V_{1}$ for $V\left(\omega_{i}\right)$ and $V_{1}\left(\omega_{i}\right)$ respectively. Starting with $\hat{D}\left(e^{j \omega_{i}}\right)$, we have

$$
\begin{aligned}
\hat{D}\left(e^{j \omega_{i}}\right) & =V^{H} \hat{P}_{n} V=V^{H} P_{s} \Delta E_{n} \Delta E_{n}^{H} P_{s} V=\beta_{i}^{H} \hat{R} E_{n} E_{n}^{H} \hat{R} \beta_{i} \\
& =\sum_{r=M^{H}+1}^{L} \beta_{i}^{H} \hat{R} S_{r} S_{r}^{H} \hat{R} \beta_{i},
\end{aligned}
$$

where $\beta_{i}=R_{s}^{+} V$. The third equality in (6) follows from theorem 1. Note that $\beta_{i}$ depends on $R_{s}$ which is dependent on the approach used to determine the covariance matrix. Therefore, the MSE of the signal zeros can be expressed as

$$
\overline{\left|\Delta z_{i}\right|_{m u}^{2}}=\left(\sum_{r=M+1}^{L} \Gamma_{\beta_{i} \beta_{i} S_{r} s_{r}}\right) /\left(V_{1}^{H} P_{n} V_{1}\right) \text {. }
$$

\subsection{Mean squared error in DOA}

Using a Taylor's series expansion of $D\left(e^{j \omega_{t}}\right)$, the mean squared error in DOA is given by

$$
\overline{\left(\Delta \omega_{i}\right)_{m u}^{2}}=\widehat{D}_{1}^{2}\left(e^{j \omega_{i}}\right) /\left\{4\left[V_{1}^{H} P_{n} V_{1}\right]^{2}\right\},
$$

where $D_{1}\left(e^{j \omega}\right)=(\partial D / \partial \omega)($ Rao \& Hari 1989b; Stoica \& Nehorai 1989). To obtain an expression for $\widehat{D}_{1}^{2}\left(e^{j \omega_{i}}\right)$, we note that

$$
\hat{D}\left(e^{j \omega}\right)=V^{H}(\omega) \hat{P}_{n} V(\omega) .
$$

Differentiating w.r.t. $\omega$, and using a first order approximation ${ }^{7}$

$$
\hat{D}_{1}\left(e^{j \omega_{i}}\right)=2 \operatorname{Re}\left(V_{1}^{H} E_{n} \Delta E_{n}^{H} V\right)=2 \operatorname{Re}\left(V_{1}^{H} E_{n}\left(P_{s} \Delta E_{n}\right)^{H} V\right) \text {. }
$$

Using the results of theorem 1 ,

$$
\hat{D}_{1}\left(e^{j \omega_{i}}\right)=-2 \operatorname{Re}\left(V_{1}^{H} P_{n} \hat{R} R_{s}^{+} V\right)=-2 \operatorname{Re}\left(\alpha_{i}^{H} \hat{R} \beta_{i}\right),
$$

where $\alpha_{i}^{H}=V_{1}^{H} P_{n}$. Squaring and using the identity $(\operatorname{Re}(x))^{2}=\frac{1}{2}\left[|x|^{2}+\operatorname{Re}(x)^{2}\right]$, we have

$$
\hat{D}_{1}^{2}\left(e^{j \omega_{i}}\right)=2\left[\left(\alpha_{i}^{H} \hat{R} \beta_{i}\right)\left(\alpha_{i}^{H} \hat{R} \beta_{i}\right)^{*}+\operatorname{Re}\left(\alpha_{i}^{H} \hat{R} \beta_{i}\right)\left(\beta_{i}^{H} \hat{R} \alpha_{i}\right)^{*}\right]
$$

\footnotetext{
${ }^{7}$ If $x=a+j b$, then $\operatorname{Re} x=a$.
} 
Taking expectation, and using theorem 1,

$$
\overline{\hat{D}_{1}^{2}}\left(e^{j \omega \omega_{i}}\right)=2\left(\Gamma_{\alpha_{i} \alpha_{i} \beta_{i} \beta_{i}}+\operatorname{Re} \Gamma_{\alpha_{i} \beta_{i} \alpha_{i} \beta_{i}}\right) .
$$

Substituting (10) in (8)

$$
\overline{\left(\Delta \omega_{i}\right)_{m u}^{2}}=\left(\Gamma_{\alpha_{i} \alpha_{i} \beta_{i} \beta_{i}}+\operatorname{Re} \Gamma_{\alpha_{i} \beta_{i} \alpha_{i} \beta_{i}}\right) /\left[2\left(V_{1}^{H}\left(\omega_{i}\right) P_{n} V_{1}\left(\omega_{i}\right)\right)^{2}\right] .
$$

Similarly, it can be shown that

$$
\overline{\left(\Delta \omega_{i} \Delta \omega_{j}\right)_{m u}}=\operatorname{Re}\left(\Gamma_{\alpha_{i} \alpha_{j} \beta_{j} \beta_{i}}+\Gamma_{\alpha_{i} \beta_{j} \alpha_{j} \beta_{i}}\right) /\left[2\left(V_{1}^{H}\left(\omega_{i}\right) P_{n} V_{1}\left(\omega_{i}\right)\right)\left(V_{1}^{H}\left(\omega_{j}\right) P_{n} V_{1}\left(\omega_{j}\right)\right)\right]
$$

\section{Analysis of the minimum-norm method}

\subsection{Background}

In the minimum-norm method (Kumaresan \& Tufts 1983), the null spectrum $D_{m n}\left(e^{j \omega}\right)$ is defined as ${ }^{8}$

$$
D_{m n}\left(e^{j \omega}\right)=\left|\left[1, g^{H}\right] V(\omega)\right|^{2} .
$$

$\left[1, g^{T}\right]^{T}$ is a vector completely in the noise subspace with the smallest Euclidean norm (Kumaresan \& Tufts 1983).

Before we do the analysis, the following minor modification is useful. Since $\left[1, g^{T}\right]^{T}$ is in the noise subspace, without loss of generality, it can be defined as one of the eigenvectors corresponding to the noise subspace, i.e. $S_{M+1}=\left[1 /\left(1+\|g\|^{2}\right)^{1 / 2}\right]\left[1, g^{T}\right]^{T}$, and the remaining eigenvectors corresponding to the noise subspace chosen accordingly.

$$
D_{m n}\left(e^{j \omega}\right)=\left|S_{M+1}^{H} V(\omega)\right|^{2}=V^{H}(\omega) P_{g} V(\omega),
$$

where $P_{g}=S_{M+1} S_{M+1}^{H}$.

\subsection{Analysis}

The minimum-norm null spectrum is a special case of the MUSiC spectrum where only one noise eigenvector is being used. The expressions for the mean squared error follow simply from the previous analysis of MUSIC by replacing $P_{n}$ with $P_{g}$. From (7) and (11), following are the expressions for the MSE in zeros and DOA.

$$
\begin{aligned}
& \overline{\left|\Delta z_{i}\right|_{m n}^{2}}=\left(\Gamma_{\beta_{i} \beta_{i} S_{M+1} S_{M+1}}\right) /\left(V_{1}^{H} P_{g} V_{1}\right) . \\
& \overline{\left(\Delta \omega_{i}\right)_{m n}^{2}}=\left[\Gamma_{v_{i} v_{i} \beta_{i} \beta_{i}}+\operatorname{Re}\left(\Gamma_{v_{i} \beta_{i} v_{i} \beta_{i}}\right)\right] / 2\left(V_{1}^{H}\left(\omega_{i}\right) P_{g} V_{1}\left(\omega_{i}\right)\right)^{2},
\end{aligned}
$$

where $v_{i}^{H}=V_{1}^{H} P_{g}$.

\footnotetext{
${ }^{8}$ Subscript $m n$ denotes quantity associated with the minimum-norm method.
} 


\section{Special case: Forward only covariance estimator}

As indicated previously, the nature of $\Gamma$ depends on the type of errors in $\hat{R}$. To illustrate how the different covariance matrices and statistical assumptions can be accommodated, the popular forward only covariance matrix estimator is considered in detail. Results when the spatially smoothed covariance estimate is used can be found in Rao \& Hari (1990b). In the forward only approach, an estimate of the covariance matrix is obtained by straightforward (time) averaging of the outer product of the $N$ snapshots, i.e.

$$
\begin{aligned}
& R_{f}=\overline{Y(n) Y^{H}(n)} \\
& \hat{R}_{f}=\frac{1}{N} \sum_{n=1}^{N} Y(n) Y^{H}(n) .
\end{aligned}
$$

To derive an explicit expression for $\Gamma_{\alpha \beta \gamma \delta}$ for the forward only covariance estimator, the following statistical assumptions on the data are made.

A1: The noise vector $N(n)$ is assumed to be a zero mean, complex white circularly Gaussian random vector, i.e. $\overline{N(n) N(n)^{H}}=\sigma_{n}^{2} I$ and $\overline{N(n) N(n)^{T}}=0$. It is also assumed to be independent of the complex signal amplitude vector $p(n)$.

A2: The complex amplitude vector $p(n)$ is modeled as a zero mean circularly Gaussian random vector.

A3: The $N$ snapshot vectors are assumed to be independent.

Under assumptions Al, A2 and A3, for the forward only approach,

$$
\Gamma_{\alpha \beta \gamma \delta}=(1 / N)\left(\alpha^{H} R_{f} \beta\right)\left(\gamma^{H} R_{f} \delta\right)
$$

Substituting (17) in the MSE expressions for MUSIC and the minimum-norm method, they can be simplified to

$$
\begin{aligned}
\overline{\left|\Delta \omega_{i}\right|_{m u}^{2}} & =\frac{\overline{\left|\Delta z_{i}\right|_{m u}^{2}}}{2(L-M)}=\frac{\sigma_{n}^{2}}{2 N} \frac{\left(\beta_{i}^{H} R_{f} \beta_{i}\right)}{V_{1}^{H} P_{n} V_{1}} \\
& =\frac{\sigma_{n}^{2}}{2 N} \frac{1}{V_{1}^{H} P_{n} V_{1}} l_{i}^{H}\left(P^{-1}+\sigma_{n}^{2} P^{-1}\left(V_{s}^{H} V_{s}\right)^{-1} P^{-1}\right) l_{i} \\
\overline{\left|\Delta \omega_{i}\right|_{m n}^{2}} & =\frac{\overline{\left|\Delta z_{i}\right|_{m n}^{2}}}{2}=\frac{\sigma_{n}^{2}}{2 N} \frac{\left(\beta_{i}^{H} R_{f} \beta_{i}\right)}{V_{1}^{H} P_{g} V_{1}} \\
& =\frac{\sigma_{n}^{2}}{2 N} \frac{1}{V_{1}^{H} P_{g} V_{1}} l_{i}^{H}\left(P^{-1}+\sigma_{n}^{2} P^{-1}\left(V_{s}^{H} V_{s}\right)^{-1} P^{-1}\right) l_{i}
\end{aligned}
$$

where $l_{i}$ is the $i$ th column of the identity matrix $l$. Comparing the above equations, the following important conclusions can be drawn.

1. Since $V_{1}^{H} P_{n} V_{1} \geqslant V_{1}^{H} P_{g} V_{1}$,

$$
\overline{\left(\Delta \omega_{i}\right)_{m u}^{2}} \leqslant \overline{\left(\Delta \omega_{i}\right)_{m n}^{2}}
$$

Therefore, the estimates obtained using MUSIC have smaller variance compared to the minimum-norm method. This provides a rigorous proof to the observations 
in Rao \& Hari (1989c). The difference is significant as the length of the array $L$ increases.

2. The spectral efficiency factor for MUSIC is $\frac{1}{2}(L-M)^{-1}$ compared to $\frac{1}{2}$ for the minimum-norm method. This suggests that using a spectral approach is disadvantageous for MUSIC. In other words, though the estimates obtained via MUSIC have a smaller variance, the peaks in the spatial spectrum may not be sharp. This loss of resolution was observed when the spectral forms were compared (Kaveh \& Barabell 1986; Jeffries \& Farrier 1988).

3. The above expressions, and conclusions for the forward only covariance estimator are also valid for any general array configuration simply by using the appropriate steering vectors. Hence, MUSIC will always perform better than the minimum-norm method even when a general array is considered.

To further explain these results, a numerical example is presented.

Example 1. A uniform linear array with $L=8, M=1, N=100, \mathrm{DOA}=18^{\circ}$ is considered.

The performance of the subspace metheds using the forward only covariance estimator is studied as a function of the signal to noise ratio (SNR) and the array length $L$. Figures 1 and 2 present the MSE in the signal zero and the DOA for the subspace methods as a function of SNR. It is observed that the results of the evaluation of the expressions based on the theory developed, matches the results obtained through the simulations quite closely. The performance of all methods improves with SNR. It is seen that as predicted by theory, the minimum-norm method has a lower MSE in signal zeros than MUSIC but has a higher MSE in DOA as compared to MUSIC. Also the performance of MUSIC is close to the Cramér-Rao bound for moderate to high values of SNR. Though ESPRIT is not analysed in this paper, it can also be analysed in a similar manner; using the framework developed in this paper. Its behaviour is similar to the minimum-norm method, and the results are shown here for purposes of comparison.

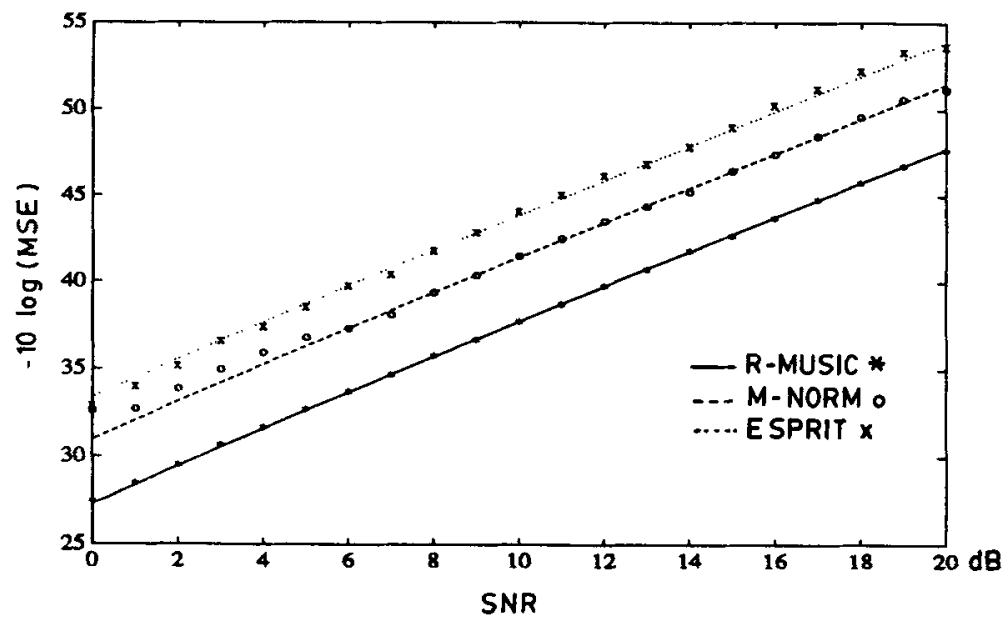

Figure 1. The theoretical and experimental mean squared error in signal zero vs SNR for Root-MUSIC, minimum-norm method and ESPRIT. The parameters used are $L=8, M=1$, $\mathrm{DOA}=18^{\circ}, N=100$. 


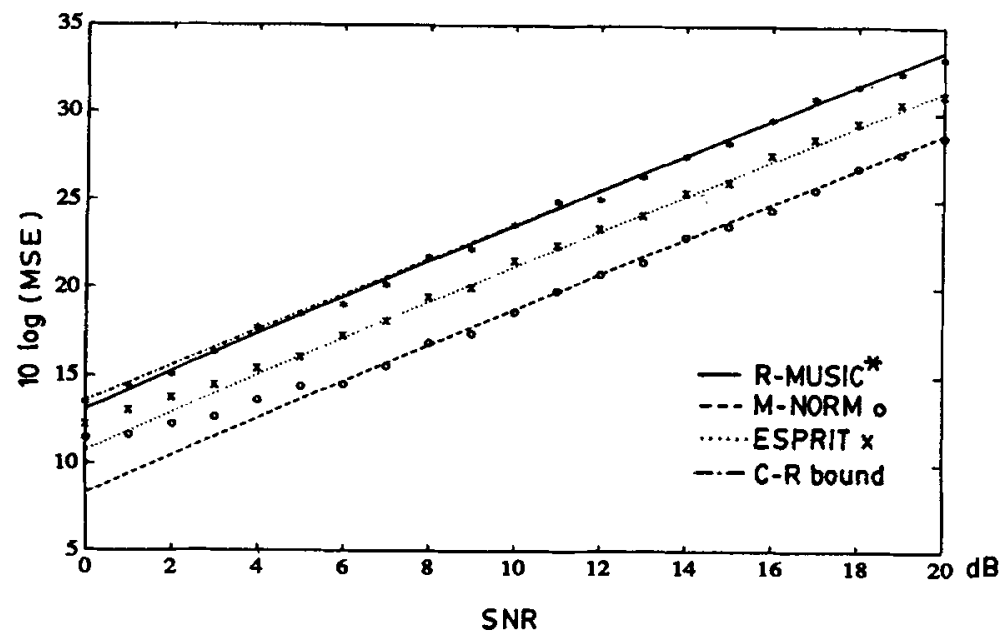

Figure 2. The theoretical and experimental mean squared error in DOA vs SNR for Root-MUSIC, minimum-norm method and ESPRIT along with the Cramér-Rao bound. The parameters used are $L=8, M=1, \mathrm{DOA}=18^{\circ}, N=100$.

Figure 3 shows the ratio of the MSE in DOA of the minimum-norm method to the MSE in DOA of MUSIC, i.e. $\overline{\left(\Delta \omega_{i}\right)_{m n}^{2}} \overline{\left(\Delta \omega_{i}\right)_{m u}^{2}}$, as a function of the array length $L$. It can be seen that the ratio increases implying that the performance of MUSIC is significantly better than that of the minimum-norm method as the array length increases.

\section{Summary}

A general framework for the analysis of eigen-based subspace methods has been developed in this paper. The framework leads to a much simpler and tractable analysis

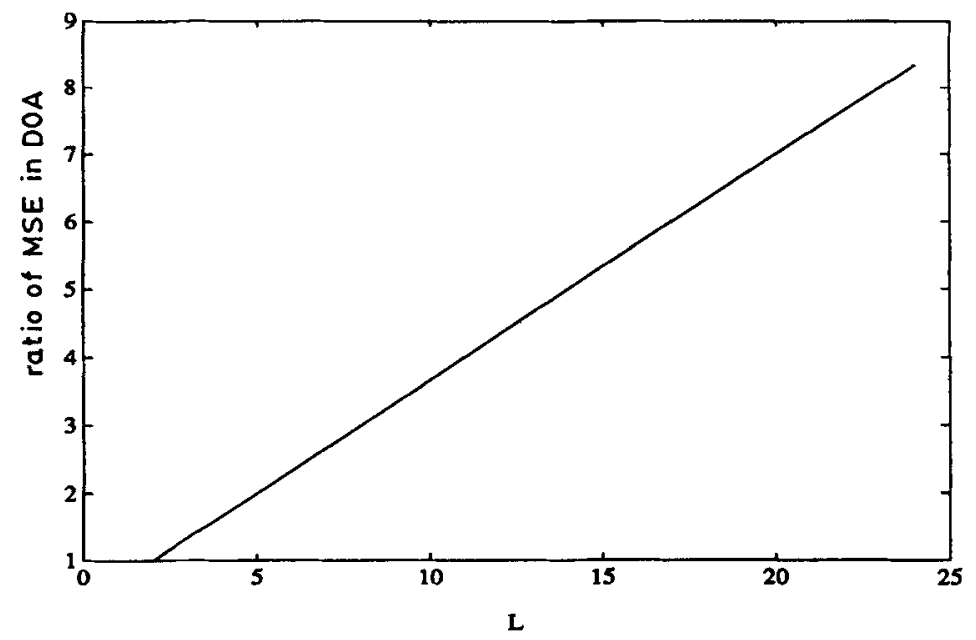

Figure 3. The ratio of the MSE in DOA of minimum-norm method to the MSE in DOA of MUSIC, as a function of the array length $L$. The parameters used are $M=1, D O A=18^{\circ}$, $N=100$, SNR $=10 \mathrm{~dB}$. 
procedure compared to procedures that start with the statistics of the eigenvectors. The overall procedure consists of two main steps. The first step relates the errors in the covariance matrix to errors in the subspaces, and the second step relates errors in the subspaces to the errors in the direction of arrival (DOA) estimates. Combining these two steps along with the statistics of the data, general expressions for the mean squared error in the DOA estimate are derived. The general results can be specialized to deal with different sources of error to obtain additional insight. The potential of the approach is demonstrated by analysing two subspace methods, MUSIC and the minimum-norm method. The general expressions for the mean squared error in the DOA are derived. The results are then specialized for the case when the forward only covariance estimator is used, and interesting insight into the relative performances of the methods is provided.

This work was supported by the US ARMY Research Office under Grant No. DAAL-0390-G-0095.

\section{References}

Bienvenu G, Kopp L 1979 Principle de la goniometrie passive adaptive. In Proc. 7'eme Colloque GRESIT Nice, France, pp. 106/1-106/10

Brillinger D R 1980 Time series: Data analysis and theory expanded edn (San Francisco: Holden-Day)

Cadzow J A 1988 A high resolution direction-of-arrival algorithm for narrow-band coherent and incoherent sources. IEEE Trans. Acoust., Speech Signal Process. ASSP-34: 965-979

Chung K L 1974 A course in probability theory (New York: Academic Press)

Clergeot H, Tressens S, Ouamri A 1989 Performance of high resolution frequencies estimation methods compared to the Cramér-Rao bounds. IEEE Trans. Acoust., Speech Signal Process. ASSP-37: 1703-1720

Friedlander B 1989 A sensitivity analysis of the MUSIC algorithm. In Proc. ICASSP 89 (New York: IEFE Press) pp. 2811-2814

Hua Y, Sarkar T K 1988 Perturbation analysis of TK method for harmonic retrieval problems. IEEE Trans. Acoust., Speech Signal Process. ASSP-36: 228-240

Jeffries D J, Farrier D R 1985 Asymptotic results for eigenvector methods. Proc. Inst. Electr. Eng. F132: 589-594

Jeffries D J, Farrier D R 1988 Theoretical performance prediction of the MUSIC algorithm. Proc. Inst. Electr. Eng. F135: 216-224

Kaveh M, Barabell A J 1986 The statistical performance of the MUSIC and the minimum-norm algorithms in resolving plane waves in noise. IEEE Trans. Acoust., Speech Signal Process. ASSP-34: 331-341

Kumaresan R, Tufts D W 1983 Estimating the angles of arrival of multiple plane waves. IEEE Trans. Aerosp. Electron. Syst. AES-19: 134-139

Hari K V S 1990 Performance analysis of subspace methods for direction of arrival (DOA) estimation, $\mathrm{Ph} \mathrm{D}$ thesis, University of California, San Diego, La Jolla, CA

Ottersten B E 1989 Parametric subspace fitting methods for array signal processing, Ph D thesis, Stanford University, Stanford

Ottersten B, Viberg M, Kailath T 1990 Asymptotic robustness of sensor array processing methods. In Proc. ICASSP (New York: IEEE Press) pp. 2635-2638

Porat B, Friedlander B 1988 Analysis of the asymptotic relative efficiency of the MUSIC algorithm. IEEE Trans. Acoust., Speech Signal Process. ASSP-36: 532-544

Pillai S U, Kwon B H 1989 Performance analysis of MUSIC-type high resolution estimators for direction finding in correlated and coherent scenes. IEEE Trans. Acoust., Speech Signal Process. 37: 1176-1189

Rao B D 1988 Lowering the threshold SNR of singular value decomposition based methods. In Proc. ICASSP (New York: IEEE Press) pp. 2472-2475

Rao B D, Hari K V S 1989a Performance analysis of ESPRIT and TAM in determining the direction of arrival of plane waves in noise. IEEE Trans. Acoust., Speech Signal Process. ASSP-37; 1990-1994 
Rao B D, Hari K V S 1989b Performance analysis of Root-MUSIC IEEE Trans. Acoust., Speech Signal Process. ASSP-37: 1939-1949

Rao B D, Hari K V S 1989c Statistical performance analysis of minimum-norm method. Proc. Inst. Electr. Eng. F136: 125-134

Rao B D, Hari K V S 1990a Effect of spatial smoothing on state-space methods/ESPRIT. In Proc. IEEE ASSP $5^{\text {th }}$ Workshop Spectrum Est. Modeling (New York: IEEE Press) pp. 377-381

Rao B D, Hari K V S $1990 \mathrm{~b}$ Effect of spatial smoothing on the performance of MUSIC and the minimum-norm method. Proc. Inst. Electr. Eng. F137: 449-458

Roy R, Kailath T 1989 ESPRIT - Estimation of Signal Parameters via Rotational Invariance Techniques. IEEE Trans. Acoust., Speech Signal Process. ASSP-37: 984-995

Schmidt R O 1979 Multiple emitter location and signal parameter estimation. In Proc. RADC Spectrum Estimation Workshop (New York: IEEE Press) pp. 243-258

Shan T J, Wax M, Kailath T 1985 On spatial smoothing for direction-of-arrival estimation of coherent signals. IEEE Trans. Acoust., Speech Signal Process. ASSP-33: 806-811

Stoica P, Nehorai A 1989 MUSIC, maximum likelihood and Cramér-Rao bound. IEEE Trans. Acoust., Speech Signal Process. ASSP-37: 720-741

Stoica P, Nehorai A 1991 Performance comparison of subspace rotation and MUSIC like methods. IEEE Trans. Acoust., Speech Signal Process. ASSP-39: 446-453

Swindlehurst A, Ottersten B, Kailath T 1989 An analysis of MUSIC and Root-MUSIC in the presence of sensor perturbations. In Proc. $23^{r d}$ Asilomar Conf. Signal, Syst., Comput. (Maple Publishers)

Viberg M 1989 Subspace fitting concepts in sensor array processing, Ph D thesis, Linköping University, Linköping, Sweden

Williams R T, Prasad S, Mahalanabis A K. Sibul L H 1988 An improved spatial smoothing technique for bearing estimation in a multipath environment. IEEE Trans. Acoust., Speech Signal Process. ASSP-36: 425-432 